\title{
Beneficios de combinar métodos para analizar causas de muertes maternas, Bucaramanga, Colombia
}

\author{
Laura Andrea Rodríguez Villamizar, ${ }^{1}$ Myriam Ruiz-Rodríguez ${ }^{1}$ \\ y María Leonor Jaime García²
}

Forma de citar Rodríguez Villamizar LA, Ruiz-Rodríguez M, Jaime García ML. Beneficios de combinar métodos para analizar causas de muertes maternas, Bucaramanga, Colombia. Rev Panam Salud Publica. 2011; 29(4):213-9.

RESUMEN Objetivo. Definir puntos críticos de cambio en el proceso de la atención materna, orientar la toma de decisiones alrededor del tema y apoyar el fortalecimiento de las políticas de prestación de servicios con miras a alcanzar el objetivo de desarrollo del milenio de mejorar la salud materna. Métodos. Estudio descriptivo retrospectivo de serie de casos de las muertes maternas registradas en Bucaramanga entre 2004 y 2009. Se estudiaron fichas epidemiológicas, historias clínicas, visitas de campo y actas de comités de análisis. Se utilizaron como métodos de análisis el camino de la supervivencia (análisis de demoras) y el de detección de eslabones críticos de la atención. Se trianguló la información obtenida.

Resultados. Las 10 muertes maternas ocurrieron en una zona urbana y contaron con la atención de profesionales en establecimientos de salud. Los cuatro tipos de demoras -en el reconocimiento del problema, en la oportunidad de la decisión y de la acción, en la atención/ logística de referencia y en la calidad de la atención-estuvieron presentes con una frecuencia similar en los 10 casos estudiados. Los eslabones críticos para la prevención de muertes maternas fueron los relacionados con fallas en la integración vertical y horizontal del proceso de atención y en su calidad.

Conclusiones. La combinación de metodologías de análisis permitió establecer las fallas de la atención que más se relacionaron con las muertes maternas. Sin embargo su uso debe acompañarse de otros métodos que permitan identificar determinantes que trasciendan el contexto de la prestación de servicios. Se documenta una lección aprendida sobre la importancia de la continuidad de las intervenciones y su monitoreo como un factor de éxito en la reducción de la mortalidad materna.

Palabras clave Mortalidad materna; salud materna; bienestar materno; servicios de salud; Colombia.

"Mejorar la salud materna" es uno de los Objetivos de Desarrollo del Milenio (ODM) con menos progreso en su consecución (1). Una de las pruebas es que en

\footnotetext{
1 Universidad Industrial de Santander, Facultad de Salud, Escuela de Medicina, Departamento de Salud Pública, Bucaramanga, Colombia. La correspondencia se debe dirigir a Laura Andrea Rodríguez Villamizar, laurarovi78@gmail.com

2 Alcaldía de Bucaramanga, Secretaría de Salud y Ambiente, Bucaramanga, Colombia.
}

los países en desarrollo las mujeres siguen muriendo por causas prevenibles relacionadas con los servicios de salud (2). De allí que en estos países el análisis de las causas de las muertes maternas, una herramienta para la planificación, evaluación y mejoramiento de los servicios de salud implementada hace ya más de una década, continúa siendo un componente fundamental de la salud pública.
En Colombia, de las diferentes metodologías que se aplican al análisis de las muertes maternas, la más utilizada es la que se conoce como "la ruta de la vida" o "análisis de las demoras" $(3,4)$. Si bien esta metodología es probablemente una de las más usadas en el contexto latinoamericano, existen otras con finalidades similares, como la introducida recientemente en México, donde se ha utilizado una herramienta de análisis denominada 
"Detección de eslabones críticos de la atención para prevenir muertes maternas", que ayuda a los comités de análisis de muerte materna y perinatal municipales a transitar desde el enfoque centrado en la fisiopatología de la paciente, al enfoque de análisis del desempeño $(5,6)$. Teniendo en cuenta que estas metodologías parten del análisis individual de las muertes se constituyen en estrategias apropiadas para uso en el nivel local o regional.

Bucaramanga, capital del departamento de Santander, tiene 530000 habitantes, con $99 \%$ de población urbana y $11,3 \%$ de sus hogares con necesidades básicas insatisfechas, y es considerada la quinta ciudad más desarrollada económicamente en Colombia. Es una ciudad reconocida en el ámbito nacional por tener 97\% de su población con cobertura de salud y $99 \%$ de atención institucional de partos. En 2007 fue galardonada por el CIFAL Atlanta (Centro Internacional para la Formación de Autoridades de Gobierno y Líderes de la Sociedad Civil) con el premio "América" a la excelencia en el servicio público, por su avances en la seguridad materna reflejados en la ausencia de muertes en este sector de población.

Sin embargo, cuatro sorpresivas muertes maternas - tres de ellas preveniblesocurridas durante el primer trimestre de 2009 alertaron a las autoridades municipales de salud y a la academia en torno a la búsqueda de soluciones para evitar nuevas muertes. La Dirección Local de Salud (DLS) analizó dichas muertes según el protocolo de vigilancia nacional que utiliza la metodología del "análisis de las demoras" y, en la última de las cuatro muertes, la Universidad Industrial de Santander (UIS) apoyó el abordaje del análisis complementando con la metodología "de eslabones críticos".

Así se encontró que esta metodología proporcionaba datos complementarios y precisos sobre omisiones o incumplimientos ocurridos en cada uno de los eslabones de atención con los que la mujer tuvo contacto (4-6). Fundamentados en este hallazgo, la DLS y la UIS realizaron el presente estudio retrospectivo de casos de muertes maternas ocurridas consecutivamente entre 2004 y 2009, cuyos objetivos fueron definir puntos críticos de la atención, orientar la toma de decisiones alrededor del tema y apoyar el fortalecimiento de las políticas de prestación de servicios con miras a alcanzar el ODM 5.

En este artículo se muestran los resultados de dicho estudio, que incluyó una combinación de metodologías de análisis de las muertes maternas y la triangulación de fuentes de información. Se comparten asimismo lecciones que ponen en evidencia la necesidad de que, una vez que las muertes maternas empiezan a disminuir, el sistema de salud redoble sus esfuerzos para asegurar su continuidad y fortalecer el monitoreo de las estrategias y programas institucionales y comunitarios dirigidos a combatir la mortalidad en este grupo de población. La intención es que estos hallazgos puedan ser aprovechados también por otros países de la Región que han puesto en marcha medidas para controlar y reducir la mortalidad materna.

\section{MATERIALES Y MÉTODOS}

Se realizó un estudio descriptivo retrospectivo aplicado a una muestra conformada por los casos de muertes maternas ocurridos en el municipio de Bucaramanga y registrados en el sistema de vigilancia en salud pública municipal entre el 1 enero de 2004 y el 31 de diciembre de 2009.

Se tomó como definición de muerte materna la establecida en el protocolo de vigilancia de la muerte materna vigente en Colombia (4). Se tuvieron en cuenta las siguientes variables de las mujeres fallecidas: edad, escolaridad, estado civil, paridad, régimen de afiliación en salud y zona de procedencia. Sobre el evento obstétrico se tuvieron en cuenta la edad gestacional o tiempo posparto al momento de la muerte, sitio o establecimiento de salud donde ocurrió la defunción, establecimiento que atendió el parto, establecimiento que atendió el control prenatal, presencia de complicaciones del embarazo, causa básica de la muerte, cadena de interacciones de cada caso con los servicios de salud y presencia y tipo de demora en la ruta de la vida.

Tanto las variables de estudio de las mujeres y del evento obstétrico como las características del contacto con los servicios de salud fueron tomadas de las fichas epidemiológicas de casos, de las historias clínicas, de los registros de las visitas de investigación del caso realizadas con el esposo o un familiar para el análisis del comité municipal de mortalidad materna y de las actas de análisis de las muertes suministradas por la DLS. Las actas mencionadas corresponden a los registros municipales de cada una de las muertes en las que, además de las au- toridades de salud, participaron las empresas aseguradoras de las mujeres, los establecimientos que les brindaron atención y especialistas en gineco-obstetricia como pares evaluadores de la mayoría de los casos. También se revisaron los documentos de la DLS para consultar acerca de las acciones que esta institución llevó a cabo cada año con el objeto de disminuir la mortalidad materna en Bucaramanga.

Para cada caso se definió la causa básica de muerte y se realizó el análisis de la ruta de la vida o análisis de demoras, de acuerdo con la guía nacional (4). Esta guía utiliza el modelo de Maine, modificado con cuatro demoras, que tiene como finalidad identificar las barreras que encuentran las mujeres para acceder y utilizar los servicios. La primera demora se refiere al reconocimiento del problema, la segunda a la oportunidad de la decisión y de la acción, la tercera al acceso a la atención/logística de referencia, y la cuarta a la calidad de la atención.

De igual forma, en cada caso se realizó la caracterización de la cadena de eslabones (interacciones) que tuvo la mujer con los servicios de atención y, en cada eslabón, se analizó si hubo omisión, demora o falta de calidad en los procedimientos técnico-científicos. Luego, con el total de los casos se ponderó la frecuencia con que se registraron incumplimientos en los eslabones críticos y se identificaron los procesos organizacionales y funcionales involucrados para detectar los factores que condicionaron o propiciaron la presencia de dichos problemas (7). Posteriormente se realizó la identificación de puntos comunes y complementarios para proponer acciones de mejora.

\section{RESULTADOS}

\section{Características de las mujeres fallecidas}

Entre enero de 2004 y diciembre de 2009 se registraron 10 muertes maternas en el municipio de Bucaramanga, todas en establecimientos de salud (figura 1). Las edades de las mujeres fallecidas oscilaron entre 22 y 37 años, con una mediana de 33 años. Siete de las 10 mujeres tenían algún grado de educación secundaria o técnica, y la mitad no tenían pareja en el momento de la muerte. En relación con su paridad, cuatro mujeres eran primerizas e igual número tenían cuatro o más hijos. La mitad de las mujeres fallecidas se dedicaban a su hogar y las que trabajaban, 
FIGURA 1. Mortalidad materna en Bucaramanga, Colombia, 2004-2009

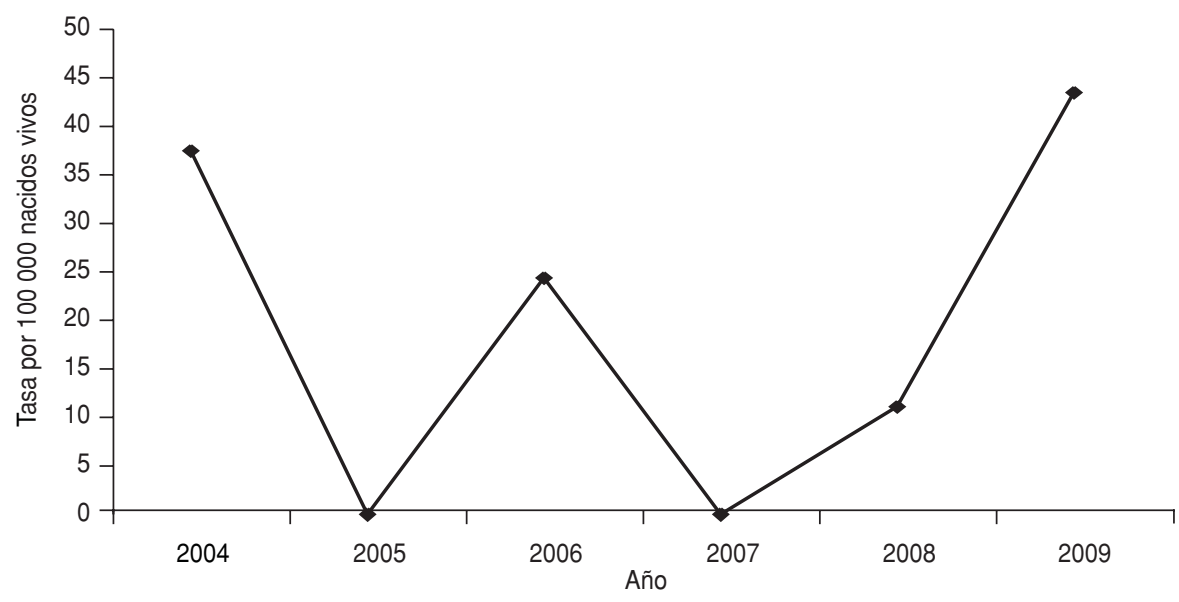

Fuente: elaboración de los autores.

excepto una, lo hacían en empleos informales. Todas las mujeres eran de nivel socioeconómico medio o bajo y una era de origen indígena.

El nivel socioeconómico de las mujeres estuvo asociado directamente con su cobertura en salud. Tres pertenecían a los estratos más bajos y estaban afiliadas al seguro vía subsidios del Estado, mientras que las demás estaban aseguradas mediante el pago de una prima o cuota. Todas las mujeres residían en la zona urbana del municipio y recibieron atención profesional en establecimientos de salud.

\section{Eventos y causas básicas de muerte}

En 2004 se registraron tres muertes ocurridas durante la gestación. El primer caso, debido a una cardiopatía congénita con hipertensión pulmonar, presentó barreras administrativas para la remisión a nivel de atención de alta complejidad y retrasos en la decisión para desembarazar a la madre. En el segundo, causado por infección por virus de inmunodeficiencia humana/síndrome de la inmunodeficiencia adquirida (VIH/sida) en fase sida C3, hubo inicio tardío de control prenatal y retrasos severos en manejo por infectología y en el inicio de terapia antirretroviral. El tercer caso fue una mujer de origen indígena también infectada por $\mathrm{VIH} /$ sida (C3), sin visitas prenatales, quien falleció tras su ingreso al servicio de urgencias al parecer en estado post-ictal.

A finales de ese año el municipio comenzó a diseñar el "Plan de choque para la reducción de la mortalidad materna en Bucaramanga", el cual siguiendo las directrices nacionales hizo hincapié en mejorar la movilización social, la calidad de la atención y la vigilancia en salud pública. El plan, desarrollado en 2005, centró sus estrategias principalmente en la capacitación a la red de prestadores de servicios sobre un modelo de control prenatal de bajo riesgo y en el fortalecimiento de los comités de análisis de mortalidad materno-perinatal, con participación de diferentes actores del sistema sanitario - academia, aseguradores, entes territoriales y prestadores. Durante ese año no se registraron muertes maternas.

En 2006 se notificaron dos muertes relacionadas con sangrado posparto debidas a dos casos se identificaron problemas de disponibilidad oportuna de hemoderivados y fallas técnicas en el manejo de la complicación, así como ausencia o inicio tardío de control prenatal. En 2007 no se informaron muertes maternas al sistema de vigilancia, pero durante ese año comienza a debilitarse la continuidad de los comités de análisis de la mortalidad materno-perinatal y la capacitación a la red prestadora de servicios.

En 2008 se registró una muerte durante el puerperio mediato en una mujer con cardiopatía congénita severa, previamente asintomática. Se identificaron fallas en la periodicidad de los controles, falta de oportunidad en el manejo por cardiología y alta precipitada en el puerperio acorde con el alto riesgo de la madre. Este año coincidió con el cambio de gobierno municipal y el ajuste de las directrices nacionales para el proceso de planificación en salud, lo cual representó retrasos en el inicio de los programas de salud materna y la discontinuidad de las dos estrategias mencionadas como bandera del Plan de Choque. atonía uterina y placenta pércreta. En los
A lo largo del primer trimestre de 2009 se notificaron cuatro muertes: tres durante el puerperio mediato y una durante la gestación. Las causas fueron: a) placenta pércreta con extensión a vejiga y peritoneo en mujer migrante con cuatro cesáreas previas quien, por barreras administrativas (no asegurada, residente habitual en otro departamento del país), no recibió ningún control prenatal; b) atonía uterina, identificándose problemas críticos como falta de unidades de sangre en el establecimiento, fallas en el proceso de remisión al tercer nivel de atención en salud, en el acceso a la unidad de cuidados intensivos e imprecisiones en procedimientos invasivos; c) neumonía complicada por errores en el manejo ambulatorio durante el puerperio, y d) cáncer invasivo de mama en una mujer que, por razones religiosas, decidió embarazarse, quien no fue atendida con el equipo multidisciplinario requerido dada su condición física y emocional.

\section{Análisis de las muertes maternas}

Análisis de la ruta de la vida o de las demoras. Se encontró que los cuatro tipos de demoras estuvieron presentes en una frecuencia similar en los casos estudiados (cuadro 1). La primera demora - el reconocimiento del problema- se evidenció por la consulta tardía a los servicios de control prenatal relacionada, principalmente, con el desconocimiento de los derechos de las gestantes, que lleva a retrasar su solicitud de servicios en el nivel básico de atención. La segunda -oportunidad de la decisión y de la acción- se dio en algunas mujeres que no expresaron una razón diferente de inicio tardío de control prenatal que su decisión propia o resolvieron quedar embarazadas pese a conocer sus riesgos bajo ciertas condiciones de salud. En la tercera demora - acceso a la atención/logística de referencia- no se encontraron dificultades relacionadas con el acceso geográfico a los servicios de salud, pero en cuatro casos sí las hubo en la logística de referencia a especialistas o niveles superiores.

Finalmente, en la cuarta demora - calidad de la atención en los establecimientos de salud - se detectaron deficiencias en relación con la clasificación de riesgo, incluido el psicosocial. En las salas de partos se evidenció poca claridad en el manejo de protocolos y falta o escasez de insumos o personal de salud. También se hallaron deficiencias en el control mé- 
dico del puerperio, especialmente en pacientes clasificadas como de alto riesgo.

Análisis de eslabones críticos. Como se puede observar en el cuadro 2, los eslabones de atención que estuvieron más comprometidos con fallas, omisiones y/o falta de apego a protocolos fueron aquellos ubicados en los servicios de menor complejidad, como los programas de salud sexual y reproductiva, el programa de control prenatal y la referencia del embarazo de alto riesgo. Con relación al primer eslabón - programas preventivos-, en todas las muertes se identificaron fallas relacionadas con los servicios de educación y consejería en planificación familiar, especialmente en las mujeres que tenían condiciones de alto riesgo basal, quienes durante todo el período de estudio representaron la mitad de los casos. En el segundo eslabón destacaron fallas en las acciones de educación e información y ausencia de demanda inducida y búsqueda activa de embarazadas por parte de las instituciones, lo cual se presenta como ausencia o inicio tardío del control prenatal.

En los eslabones tercero y cuarto fue común encontrar falta de remisión oportuna. En los servicios de atención de parto se identificaron dos problemas críticos: falta de insumos y errores en el manejo de las complicaciones. Los servicios de atención en el puerperio, por otro lado, revelaron fallas relacionadas con el alta temprana de la paciente y ausencia de seguimiento médico posterior.

Convergencia de los dos métodos de análisis. En el cuadro 3 se presenta la intersección de las causas relacionadas con las demoras y los eslabones de la atención, demostrándose que en ambos - y en todos los casos - la calidad de las acciones tiene una influencia muy importante en los pasos subsecuentes de la atención. Se observó que, aun cuando seis de las muertes ocurrieron durante el puerperio y una durante el parto, hubo una serie de omisiones en el proceso previo de atención que fueron críticas para los desenlaces. El análisis de los dos métodos permitió identificar más claramente los problemas de la calidad de la atención en cada uno de los puntos de encuentro entre la gestante y el sistema de salud. Sin embargo, si se tienen en cuenta las causas directas de las muertes, la calidad de la atención del parto y el control del puerperio aparecen como los eslabones más críticos en el proceso de atención, debido muy probablemente a las condiciones de alto riesgo basal que presentaron la mitad de las mujeres.

\section{DISCUSIÓN}

En este trabajo se analizaron los casos de muertes maternas en la ciudad de Bucaramanga durante un período de seis años, utilizando la combinación de dos metodologías complementarias. Mediante el análisis de las demoras se pudo determinar que en 6 de los 10 casos de muerte registrados hubo fallas relacionadas con el acceso y la periodicidad de la atención durante el embarazo, así como con retrasos en las decisiones médicas apropiadas para cada situación específica.

Desde esta perspectiva de análisis, se sugiere que las demoras en la atención están relacionadas con el desconocimiento de derechos en salud, la falta de reconocimiento de riesgos personales por parte de las gestantes y la calidad de la atención en casi todos los puntos de la atención materna. Es posible que la dinámica del sistema de salud actual, donde la educación en derechos y el fomento de la participación ciudadana no se reconocen como ejes fundamentales de la prestación de servicios y donde la atención no está centrada en las necesidades de las personas sino en las del mercado, pueda tener alguna responsabilidad en estos hallazgos que finalmente se traducen en obstáculos para el camino de la supervivencia de algunas gestantes. Estudios como el de Ruiz y colaboradores señalan las consecuencias del modelo de mercado del sistema de salud colombiano sobre la atención materna, en tanto otros (como Carrillo) postulan que las altas tasas de mortalidad materna en el país están relacionadas con la falta de garantías por parte del Estado acerca del derecho a la salud de las colombianas $(8,9)$. Colombia, 2004-2009

\begin{tabular}{|c|c|c|c|c|}
\hline \multirow[b]{2}{*}{ Año/caso } & \multicolumn{4}{|c|}{ Demoras } \\
\hline & $\begin{array}{c}1 \\
\text { Reconocimiento } \\
\text { del problema }\end{array}$ & $\begin{array}{c}2 \\
\text { Oportunidad } \\
\text { en decisión } \\
\text { y acción }\end{array}$ & $\begin{array}{c}3 \\
\text { Acceso a la } \\
\text { atención/logística } \\
\text { de referencia }\end{array}$ & $\begin{array}{c}4 \\
\text { Calidad } \\
\text { de la } \\
\text { atención }\end{array}$ \\
\hline 2004-1 & & $x$ & $X$ & \\
\hline 2004-2 & $x$ & $x$ & & $X$ \\
\hline 2004-3 & $x$ & $X$ & & \\
\hline $2006-1$ & & & & $x$ \\
\hline $2006-2$ & $x$ & $x$ & & \\
\hline $2008-1$ & $x$ & $x$ & $X$ & $x$ \\
\hline $2009-1$ & $x$ & & $X$ & \\
\hline 2009-2 & $X$ & & $X$ & $X$ \\
\hline 2009-3 & & & $X$ & $x$ \\
\hline $2009-4$ & & $X$ & $x$ & $x$ \\
\hline
\end{tabular}

Fuente: elaboración de los autores.

CUADRO 2. Eslabones de atención críticos para la prevención de muertes maternas, Bucaramanga, Colombia, 2004-2009

\begin{tabular}{|c|c|c|c|c|c|c|}
\hline \multirow[b]{2}{*}{ Año/caso } & \multicolumn{6}{|c|}{ Eslabones } \\
\hline & $\begin{array}{c}1 \\
\text { Programas } \\
\text { preventivos }\end{array}$ & $\begin{array}{c}2 \\
\text { Control } \\
\text { prenatal }\end{array}$ & $\begin{array}{c}3 \\
\text { Referencia } \\
\text { alto riesgo }\end{array}$ & $\begin{array}{c}4 \\
\text { Atención } \\
2^{\circ} \text { nivel }\end{array}$ & $\begin{array}{c}5 \\
\text { Parto y } \\
\text { urgencias }\end{array}$ & $\begin{array}{c}6 \\
\text { Puerperio }\end{array}$ \\
\hline 2004-1 & $x$ & & $X$ & & & $x$ \\
\hline 2004-2 & $x$ & $X$ & $X$ & & & \\
\hline 2004-3 & $X$ & $x$ & & & & \\
\hline 2006-1 & & & & & & $X$ \\
\hline $2006-2$ & $X$ & $X$ & & & & \\
\hline 2008-1 & $X$ & $X$ & $X$ & & $X$ & $x$ \\
\hline 2009-1 & $X$ & $X$ & & & $x$ & \\
\hline 2009-2 & & $X$ & $x$ & & $X$ & \\
\hline $2009-3$ & & & $x$ & & & $x$ \\
\hline 2009-4 & $X$ & $X$ & $X$ & & & \\
\hline
\end{tabular}

Fuente: elaboración de los autores. 
CUADRO 3. Puntos débiles en la calidad de atención en salud encontrados en el análisis de causas de muertes maternas, por tipo de demora y de eslabón crítico, Bucaramanga, Colombia, 2004-2009

\begin{tabular}{|c|c|c|c|c|c|c|}
\hline \multirow[b]{2}{*}{ Demora } & \multicolumn{6}{|c|}{ Eslabones } \\
\hline & $\begin{array}{c}1 \\
\text { Programas } \\
\text { preventivos }\end{array}$ & $\begin{array}{c}2 \\
\text { Control } \\
\text { prenatal }\end{array}$ & $\begin{array}{c}\text { 3 } \\
\text { Referencia } \\
\text { alto riesgo }\end{array}$ & $\begin{array}{c}4 \\
\text { Atención } \\
2^{\circ} \text { nivel }\end{array}$ & $\begin{array}{c}5 \\
\text { Parto y } \\
\text { urgencias }\end{array}$ & $\begin{array}{c}6 \\
\text { Puerperio }\end{array}$ \\
\hline $\begin{array}{l}\text { (1) Reconocimiento del } \\
\text { problema }\end{array}$ & $\begin{array}{l}\text { Conocimiento y } \\
\text { percepción de } \\
\text { riesgos de la } \\
\text { gestación }\end{array}$ & & & & & \\
\hline $\begin{array}{l}\text { (2) Oportunidad en decisión } \\
\text { y acción }\end{array}$ & & $\begin{array}{l}\text { Conocimiento de } \\
\text { derechos de } \\
\text { las gestantes/ } \\
\text { Ausencia de } \\
\text { demanda inducida }\end{array}$ & & & & \\
\hline $\begin{array}{l}\text { (3) Acceso a la atención/ } \\
\text { logística de referencia }\end{array}$ & & & $\begin{array}{l}\text { Logística de } \\
\text { referencia a } \\
\text { niveles superiores/ } \\
\text { Demoras en la } \\
\text { atención por } \\
\text { especialistas }\end{array}$ & & & \\
\hline (4) Calidad de la atención & $\begin{array}{l}\text { Consejería en } \\
\text { planificación } \\
\text { familiar }\end{array}$ & $\begin{array}{l}\text { Clasificación de } \\
\text { riesgo/Educación } \\
\text { a gestante }\end{array}$ & & $\begin{array}{l}\text { Insumos para la } \\
\text { atención de } \\
\text { complicaciones } \\
\text { (hemoderivados) }\end{array}$ & $\begin{array}{l}\text { Manejo de } \\
\text { hemorragia } \\
\text { posparto }\end{array}$ & $\begin{array}{l}\text { Control de } \\
\text { puerperio }\end{array}$ \\
\hline
\end{tabular}

Fuente: elaboración de los autores.

Nota: en todos estos factores y áreas se encontraron diversas deficiencias, que van desde la ausencia o insuficiencia de conocimiento (p.ej. en relación a los derechos de las gestantes) hasta fallas en los distintos procesos y procedimientos y carencia de insumos (p.ej. de unidades de sangre).

Desde el análisis de los eslabones críticos se privilegió una mirada integradora del conjunto de acciones que se prestan/ omiten en cada caso y en general. Así, puesta esta mirada en cada una de las etapas del proceso (longitudinalidad de la atención), se pudo determinar que los problemas relacionados con la calidad del control prenatal y del puerperio son los que presentan mayores dificultades, así como identificar opciones de mejora en la articulación horizontal y vertical dentro de la red de atención, sobre todo en la remisión a niveles de mayor complejidad, donde el análisis reveló fallas severas.

Otro punto que fue significativo desde el análisis de los eslabones críticos fue la identificación de que una maternidad segura depende de una buena atención antes del embarazo; más de la mitad de las mujeres tenían situaciones de alto riesgo basal; en consecuencia, una adecuada consejería en planificación familiar hubiera ayudado a prevenir estas muertes. Respecto a dicho hallazgo, que valga mencionarlo no hubiera sido posible identificarlo a partir de la metodología de las demoras, distintos estudios aportan evidencia de cómo la atención antes del embarazo y la de los eslabones iniciales del modelo son los que se relacionan de manera más estrecha con tasas bajas de MM (3, 10-13). El uso conjunto de los dos métodos de análisis - y en particular el examen de la longitudinalidad de la atención - permitió al equipo investigador identificar puntos de inflexión en la política de atención materna en el municipio. Así, se puso de manifiesto que durante 2008 y 2009 se debilitó el sistema de monitoreo y se perdió la continuidad de las estrategias del Plan de Choque, contribuyendo a incrementar no solo las muertes maternas en el primer trimestre de 2009, sino también los problemas de calidad de la atención durante el parto y puerperio, muchos de los cuales retornaron a los niveles experimentados en 2006. Por todo ello sería necesario diseñar - y asegurar su sostenimiento- acciones de auditoría orientadas a la calidad como condición indispensable para garantizar resultados óptimos en salud (14).

Los resultados también revelaron que durante todo el período del estudio se mantuvieron constantes algunas prioridades de intervención escogidas en 2004, como por ejemplo la capacitación del personal de establecimientos públicos en atención prenatal de bajo riesgo, pese a que los casos de muerte materna notificados después de 2005 correspondieron en igual proporción a pacientes atendidas en establecimientos privados (régimen contributivo) y públicos, mostrando un aumento de casos con riesgo basal y obstétrico alto.

La capacitación dirigida únicamente a la atención prenatal de bajo riesgo pudo haber ocasionado descuidos en el trabajo de supervisión y capacitación en áreas clave de los programas de promoción de la salud sexual y reproductiva y de la atención de parto y puerperio, las cuales en el presente estudio aparecieron como eslabones críticos que explican varias de las causas directas e indirectas de las muertes recientes.

Es posible que estas consideraciones expliquen la ligera diferencia que se halló entre el panorama de causas de muerte materna del municipio y los correspondientes al departamento y al país. En Colombia, por ejemplo, las causas directas de muertes maternas guardan relación con las notificadas a nivel mundial y se han mantenido estables en la última década (toxemia 35\%, complicaciones del parto $25 \%$, aborto $16 \%$, otras complicaciones $9 \%$, complicaciones del puerperio $8 \%$ y hemorragias $7 \%$ ), mientras que en el departamento de Santander entre 2004 y 2007 las causas relacionadas con enfermedades infecciosas (30\%) superaron a la toxemia $(22 \%)$ y a las complicaciones del parto (20\%) (15-18). En el presente estudio, por su parte, se observó que en el municipio de Bucaramanga predominaron las muertes maternas relacionadas con complicaciones del puerperio y hemorragias posparto, y que en el período estudiado hubo ausencia de muertes por toxemia en el embarazo.

Este perfil local de mortalidad materna caracterizado por una creciente participa- 
ción de las causas relacionadas con hemorragias obstétricas es similar al informado por el departamento de Antioquia en 2004 y 2005 (19), cuando casi la mitad de las muertes maternas ocurrieron por estas causas. A la luz del presente estudio, esta situación podría deberse a que en el camino del mejoramiento de la calidad del control prenatal se ha descuidado el proceso de atención de parto y puerperio, o a la eventual debilidad de los actuales protocolos de manejo en relación con estos dos procesos de atención, si se tiene en cuenta que la guía de atención de parto está dirigida casi en su totalidad a la atención del recién nacido y en el caso de la atención de puerperio no existe una guía unificada nacional.

En conclusión, puede afirmarse que la fortaleza del análisis de las demoras es que va más allá de las causas médicas para observar cada caso en su contexto económico, social y cultural, trascendiendo la búsqueda del dato clínico o demográfico puntual y convirtiéndose en información valiosa para imprimir más efectividad a las estrategias de prevención (20). El análisis de eslabones críticos, por otro lado, permite evaluar la secuencia de procesos, desde el abordaje clínico a la organización de los servicios y a la continuidad de la atención entre los establecimientos. En el presente trabajo, el análisis de eslabones reveló una situación crítica reflejada en las fallas encontradas en los procesos de referencia de algunas mujeres, en coincidencia con las notificadas en México durante la implementación de este tipo de análisis, las cuales ya comenzaron a resolverse de manera que algunos estados lograron reducir hasta $30 \%$ de la mortalidad solamente con la intervención en este eslabón (6).

Este estudio mostró cómo la combinación de dos metodologías de análisis y de fuentes de información hace posible jerarquizar los servicios de acuerdo a su proximidad con respecto a las condiciones que ponen en peligro la vida materna. Tal jerarquización es útil por dos razones: permite identificar en cada nivel las intervenciones cruciales y ayuda a las autoridades de salud a ser selectivas en cuanto a las medidas necesarias en cada nivel para reducir significativamente las tasas de mortalidad materna.

Vale destacar que el carácter retrospectivo del estudio limitó la obtención de información a las fuentes secundarias disponibles. De esta manera, en algunas ocasiones el análisis de categorías de la metodología de eslabones tuvo que ser reconstruido a partir de varias fuentes porque, a diferencia de la metodología de las demoras, no había sido utilizada previamente en los registros de análisis individuales de casos. Los posibles sesgos de análisis que podrían haber derivado de esta limitación fueron evitados mediante la revisión de todos los registros clínicos y de las entrevistas personales disponibles para cada caso.

Una limitación fundamental identificada en ambos métodos de análisis fue la falta de inclusión de otras variables diferentes al contacto con los servicios de salud como posibles puntos críticos del proceso de las muertes maternas. Ambos enfoques centran su atención en el análisis individual de casos con especial énfasis en la interacción salud materna-servicios de salud, pero dejan de lado aspectos determinantes más distales como son el sistema de salud, las políticas públicas implementadas y su funcionamiento en el territorio. En el caso de Bucaramanga, la reconstrucción año a año del avance o retroceso de las estrategias de prevención de mortalidad materna permite sugerir que dicho factor pudo ser clave para el comportamiento errático de la muerte materna, aunque tal probabilidad no se logró probar mediante ninguno de los dos métodos.

A la luz de los resultados, se perciben algunas lecciones aprendidas que pueden ser útiles para otros países de la Región que están implementando medidas para disminuir la mortalidad materna:

1. Jerarquizar los servicios de atención obstétrica de acuerdo a su proximidad con respecto a las condiciones que ponen en peligro la salud materna permite que los tomadores de decisiones sean selectivos en cuanto a las medidas que, en cada nivel de atención, pueden impactar significativamente en la tasa de mortalidad de este grupo de población. Tal jerarquización es fácilmente visible cuando se utiliza la triangulación de metodologías y de fuentes de información.

2. Cuando un sistema de salud está mejorando sus resultados en la reducción de las muertes maternas es necesario hacer mayores esfuerzos técnicos y administrativos, especialmente durante el período en que estos eventos ocurren menos o no ocurren, para procurar que las intervenciones seleccionadas se mantengan en forma continua, a largo plazo, e incluyendo a los distintos actores que intervienen en la atención materna - hayan o no participado en un caso de muerte. Estas intervenciones deben ir acompañadas de un fuerte componente de monitoreo que garantice un sistema de atención proactivo y no reactivo a las fallas que ocurren en los distintos puntos en que una mujer se pone (u omite hacerlo) en contacto con los servicios de salud.

3. Las autoridades municipales de salud deben priorizar a las mujeres con riesgo basal alto para embarazo, asegurándose de que sean captadas por el sistema para que reciban una consejería efectiva por parte de los servicios de salud sexual y reproductiva. En el presente estudio se encontró esta falla en la mayoría de los casos analizados, de lo que se infiere que las acciones dirigidas a este punto podrían tener un fuerte impacto en la reducción de muertes maternas.

4. El apego a los protocolos debe ser una prioridad en el monitoreo que las autoridades de salud hacen al proceso de atención materno. En este sentido, hace falta contar de manera continua con un programa estructurado de capacitación a los recursos humanos y de mejoramiento de la capacidad instalada de los servicios esenciales de obstetricia.

5. Cuando se ha alcanzado un nivel adecuado de cobertura de control prenatal y de parto institucional, es necesario buscar metodologías de análisis que trasciendan el énfasis sobre el acceso y den paso a la identificación de problemas críticos en las distintas fases del proceso de atención.

6. La alianza entre los servicios y la academia permite visualizar problemas sistemáticos del proceso de atención, lo cual se convierte en un insumo muy importante para que los tomadores de decisiones actúen sobre los puntos críticos de dicho proceso.

Agradecimientos. Las autoras expresan su gratitud a los siguientes estudiantes de Medicina de la Universidad Industrial de Santander que apoyaron la recolección de información durante este trabajo: Dania Vanessa Carreño Barrera, Juan Carlos Díaz Cruz, Andrea Jurado Abril, Saúl Enrique Martínez Castañeda, Andrey Antonio Perilla Plata y María Alejandra Velásquez Sandoval. 


\section{REFERENCIAS}

1. Organización Panamericana de la Salud. Los Objetivos de Desarrollo del Milenio en las Américas. Boletín Epidemiológico. 2004;25(2): 1-15.

2. Betrán A, Wojdyla D, Posner S, Gülmezoglu AM. National estimates for maternal mortality: an analysis based on the WHO systematic review of maternal mortality and morbidity. BMC Public Health. 2005;5:131-42.

3. Maine D, Akalin M, Ward B, Kamara A. The design and evaluation of maternal mortality programs. New York: Columbia University; 1997. P. 7.

4. Ministerio de la Protección Social, Instituto Nacional de Salud, Colombia. Protocolo de vigilancia de la mortalidad materna. Colombia: Ministerio de la Protección Social; 2009.

5. Nuñez RM, Duarte MB, Javier L, Arroyo L. Más allá de las cifras: Detección de eslabones críticos en los procesos de atención para prevenir muertes maternas. Género y Salud en Cifras. 2005;3(2/3):5-15.

6. Nuñez-Urquiza RM. Utilización del método de detección de eslabones críticos de los sistemas de atención de la salud para evitar muertes maternas. Estado mundial de la infancia 2009. Salud materna y neonatal. Ginebra: UNICEF; 2009.

7. Núñez RM, Arroyo L, Martínez JA, Javier L. Guía para la detección de eslabones críticos en los procesos de atención para prevenir muertes maternas. México D.F.: Cruzada nacional por la calidad en salud; 2005.
8. Ruiz M, Wirtz V, Nigenda G. Organizational elements of health services related to maternal mortality: the cases of Chile and Colombia. Health Policy. 2009;90:149-55.

9. Carrillo A. Mortalidad materna en Colombia: reflexiones en torno a la situación y la política pública durante la última década. Rev Cienc Salud. 2007;5(2):72-85.

10. Guzmán N. Hacia una nueva política de salud en Colombia. 2. ${ }^{\text {a }}$ edición. Cali: Feriva Editores; 1992.

11. Osorio S, Guzmán N. Control prenatal y su impacto en la mortalidad materna. Análisis de una tendencia, 1994-2004, Cali, Colombia. Rev Colomb Obstet Ginecol. 2009;60(1):12-8.

12. Bulatao R, Ross J. Which health services reduce maternal mortality? Evidence from ratings of maternal health services. Trop Med Int Health. 2003;8(8):710-21.

13. Kidney E, Winter H, Khan K, Gülmezoglu M, Meads C, Deeks J, et al. Systematic review of effect of community-level interventions to reduce maternal mortality. BMC Pregnancy Childbirth. 2009; 9:2. Disponible en: http:// www.biomedcentral.com/1471-2393/9/2 Acceso el 28 de diciembre de 2010.

14. Legros S, Massoud R, Urroz O. The chilean legacies in health care quality. Int J Qual Health Care. 2002;14 supl 1:83-8

15. Ruiz Salguero M. Población, pobreza y desarrollo. Memorias del día mundial de la población 2004, Volumen 1. Colombia: Universidad Externado de Colombia; 2005.
16. Ministerio de la Protección Social de Colombia, Organización Panamericana de la Salud. Situación de salud en Colombia: Indicadores básicos 2003, 2004, 2006, 2007.

17. Ministerio de Salud, Grupo de vigilancia en salud pública, Colombia. Panorama de la mortalidad materna en Colombia 1995-2000: Logros, fracasos, compromisos y retos. Informe ejecutivo semanal Sivigila, septiembre 9-15, 2001. Colombia: Ministerio de Salud; 2001.

18. Ortiz G, Rodríguez L. Mortalidad materna en Santander 2004-2007. Informe Epidemiológico de Santander. 2007;41-7.

19. Gómez J, Vélez G, Zuleta J. La mortalidad materna en el departamento de Antioquia años 2004 y 2005. En: Departamento de Antioquia, Dirección Seccional de Salud de Antioquia (DSSA). La salud de las madres en Antioquia: un reto, un derecho, un compromiso. Medellín: DSSA; 2007. P. 264.

20. Vélez G, Gallego L, Jaramillo D. Modelo de análisis de la muerte materna: camino a la supervivencia. En: Alcaldía de Medellín, Secretaría de Salud. Modelos para el análisis de la mortalidad materna y perinatal. Medellín: Secretaría de Salud; 2005. P. 139.

Manuscrito recibido el 18 de mayo de 2010. Aceptado para publicación, tras revisión, el 10 de noviembre de 2010.

ABSTRACT Objective. Define critical points of change in the maternal care process, guide decision-making in this area, and support the strengthening of service delivery policies, with a view to achieving the Millennium Development Goal of improving maternal health.

\section{Benefits of combining methods to analyze the causes of maternal mortality, Bucaramanga, Colombia}

Methods. Retrospective descriptive study of a series of cases of maternal deaths recorded in Bucaramanga between 2004 and 2009. The study examined epidemiologic reporting cards, clinical histories, field visits, and the records of analysis committees. The road to survival (analysis of delays) and detection of critical links in care were used as the methods of analysis. The information obtained was triangulated.

Results. The 10 maternal deaths occurred in an urban area, and the women had received medical care from professionals at a health facility. The four types of delaysin recognizing the problem, in the timeliness of decisions and actions, in care/the logistics of referral, and in the quality of care-occurred with similar frequency in the 10 cases studied. The critical links in the prevention of maternal deaths were those related to deficiencies in the vertical and horizontal integration of the care process and the quality of care.

Conclusions. Combining analysis methods made it possible to identify the deficiencies in care most related to maternal deaths. However, their use should be accompanied by other methods that make it possible to identify determinants that go beyond the context of service delivery. One documented lesson learned is the importance of the continuity and monitoring of interventions as a success factor in reducing maternal mortality.

Key words Maternal mortality; maternal health; maternal welfare; health services; Colombia. 